\title{
INICIATIVA "REHAB: + CALIDAD EN TU VIVIENDA". CÓMO INVOLUCRAR AL CIUDADANO EN EL PROCESO DE MEJORA DE LA CALIDAD DE SU VIVIENDA
}

\author{
Carmen Subirón Rodrigo \\ Isabel de los Ríos Rupérez \\ Instituto Valenciano de la Edificación (IVE)
}

Artículo Recibido: 07/08/2015

Artículo Aceptado: 04/09/2015

\section{Resumen}

El presente artículo presenta la experiencia desarrollada por el Instituto Valenciano de la Edificación (IVE) en la Comunidad Valenciana, en el ámbito de la información y educación de los ciudadanos, con el objeto de impulsar la participación activa de los mismos como demandantes de calidad en las viviendas.

Hay un amplio consenso en el sector sobre la necesidad de fomentar la inversión en rehabilitación y la mejora del extenso parque de viviendas existentes. La mayoría de las viviendas son anteriores a 1980 y fueron construidas con niveles de calidad muy inferiores a los actuales. Si a esto le sumamos la ausencia de conservación y rehabilitación de las viviendas, nos encontramos con un envejecimiento prematuro de las mismas.

Mantener y rehabilitar adecuadamente la vivienda permite mejorar su calidad, pero para ello es fundamental la participación activa del usuario, que sólo será posible si cuenta con la información suficiente sobre los aspectos técnicos más relevantes a tener en cuenta. A través de la información, los usuarios pueden demandar soluciones que reduzcan los consumos energéticos, minimicen el impacto medioambiental, reduzcan el consumo de agua, mejoren el confort acústico y la accesibilidad, incorporen energías renovables, etc. Además, les permitirá tomar las decisiones adecuadas en cuanto al adecuado uso y mantenimiento de sus inmuebles, y a la hora de abordar una reforma o rehabilitación.

En 2013 se puso en marcha, por la Generalitat Valenciana, la iniciativa "REHAB: + Calidad en tu Vivienda", desarrollada por el IVE, dirigida de forma "innovadora" a involucrar al ciudadano en el proceso de mejora continua de la calidad en su vivienda.

WPS RI-SHUR, n2, 2015, vol.1, ISSN: 2387-1768 


\section{WPSReview International on Sustainable \\ Housing and Urban Renewal (RI-SHUR)}

Esta iniciativa aporta al ciudadano información detallada sobre los beneficios de invertir en el mantenimiento y la rehabilitación de sus viviendas, por qué rehabilitar, cómo hacerlo y cuáles son los resultados. Se pretende que sean los usuarios, a través de sus decisiones, los que promuevan obras de conservación y de rehabilitación, y quienes obliguen al sector de la edificación a mejorar la calidad de sus productos.

En el marco de esta iniciativa se ha desarrollado material informativo y divulgativo que se distribuye fundamentalmente a través de la web www.calidadentuvivienda.es. Así mismo, se están estableciendo convenios con entidades relacionadas, como son ayuntamientos, asociaciones ciudadanas, agentes de la propiedad, administradores de fincas, etc., con el fin de alcanzar el más alto nivel de difusión entre los ciudadanos.

\section{Abstract}

This article presents the experience gained by the Valencia Institute of Building (IVE) in the Valencian Community, in the information and education field of citizens, in order to improve the quality in their homes.

All industry players agree with the need to encourage investment in rehabilitation and improvement of existing housing. Most homes were built before 1980, with levels well below the current quality. This, coupled with the absence of conservation and rehabilitation, produces a premature aging of the housings.

Maintain and rehabilitate housing improves quality, but it is essential the active participation of the users, which is only possible if they have enough technical information about the most important aspects to consider. Through information, users may demand solutions that reduce energy consumption, minimize environmental impact, reduce water consumption, improve the acoustic comfort and accessibility, incorporating renewable energy, etc... Also, this allow them to make appropriate decisions regarding the proper use and maintenance of their property, and addressing reform or rehabilitation.

In 2013 was launched by the Valencian Autonomic Government, "REHAB: + Quality in your Home", initiative developed by the IVE, directed so "innovative" to involve citizens in the process of continuous improvement of quality in your home.

WPS RI-SHUR, n2, 2015, vol.1, ISSN: 2387-1768 


\section{WPSReview International on Sustainable \\ Housing and Urban Renewal (RI-SHUR)}

This initiative gives citizens information about the benefits of investing in the maintenance and rehabilitation of their homes, why rehabilitate, how and what the results are. Users should be, through their decisions, the promoters to the conservation and rehabilitation. They must to require to building sector to improve the quality of their products.

As part of this initiative, has been developed information and communication material, which is distributed primarily through a website www.calidadentuvivienda.es. Likewise, they are establishing agreements with related entities, such as local governments of municipalities, civic associations, real estate agents, property managers, etc., in order to achieve the highest level of diffusion among citizens.

Palabras clave: consumidor, ciudadano; vivienda; calidad; rehabilitación; energía

Área temática: Actuaciones sostenibles en la edificación / Eficiencia energética en edificación y rehabilitación

\section{Introducción}

Sin duda, la vivienda es un bien material muy importante en la vida de un ciudadano, al que mayores recursos dedican3, y que cuenta como indicador de la calidad de vida4 de las personas. Por este motivo, entendemos que es importante mejorar el conocimiento de los usuarios sobre los distintos aspectos relacionados con la calidad en la vivienda.

Cuando se va a adquirir un vehículo $u$ otro bien de consumo, es fácil encontrar información en revistas especializadas o en distribuidores, sobre cuáles son las características principales, de velocidad, o de consumo, o cuales son las ventajas o inconvenientes de uno u otro producto. En cambio, en la inversión más importante en la vida de una familia, como es una vivienda, la información de calidad es prácticamente inexistente. En la actualidad, la información se suele referir a características aparentes, tales como el aspecto de la cocina o del portal del edificio $u$ otras que no son relevantes para la calidad real del edificio.

3 INE Instituto Nacional de Estadística. Encuesta de Presupuestos Familiares, año 2012: Gasto medio por hogar y distribución porcentual por grupos de gasto.

4 Boletín informativo del Instituto Nacional de Estadística. Calidad de vida

WPS RI-SHUR, nำ2, 2015, vol.1, ISSN: 2387-1768 


\title{
WPSReview International on Sustainable
}

\author{
Housing and Urban Renewal (RI-SHUR)
}

\section{Es difícil que los usuarios adquirieran una cultura de calidad si la información relevante no se pone a su alcance.}

Por otro lado, en el sector de la edificación existente, hay un amplio consenso sobre la necesidad de fomentar la inversión en rehabilitación y la mejora del extenso parque de viviendas. A la hora de abordar de plantear estrategias, es importante tener en cuenta las directrices que nos llegan desde Europa.

En cuanto a la eficiencia energética el parque inmobiliario existente constituye el sector con mayor potencial de ahorro de energía5 y los Estados miembros deben establecer estrategias a largo plazo, más allá de 2020, para movilizar inversiones que permitan mejorar el rendimiento energético de dicho parque a partir de la renovación de sus edificios residenciales y comerciales. Las políticas europeas de eficiencia energética materializadas en la última Directiva 2012/27/UE 6 , establecen unas obligaciones a los estados miembros que pasan obligatoriamente por la rehabilitación energética.

En cuanto a la accesibilidad de los edificios y viviendas, la Ley 26/2011, de 1 de agosto7, de adaptación normativa a la Convención Internacional sobre los Derechos de las Personas con Discapacidad8, exige la realización de los ajustes razonables en materia de accesibilidad universal estableciendo incluso un plazo, que finaliza en el año 2015, momento a partir del cual pueden ser legalmente exigidos, tanto para los edificios, como para los espacios públicos urbanizados existentes y, por tanto, también controlados por la Administración Pública competente.

5 Apartado (17) de las consideraciones iniciales de la DIRECTIVA 2012/27/UE del Parlamento Europeo y del Consejo de 25 de octubre de 2012 relativa a la eficiencia energética, por la que se modifican las Directivas 2009/125/CE y 2010/30/UE, y por la que se derogan las Directivas 2004/8/CE y 2006/32/CE.

6 DIRECTIVA 2012/27/UE del Parlamento Europeo y del Consejo de 25 de octubre de 2012 relativa a la eficiencia energética, por la que se modifican las Directivas 2009/125/CE y 2010/30/UE, y por la que se derogan las Directivas 2004/8/CE y 2006/32/CE.

7 Ley 26/2011, de 1 de agosto, de adaptación normativa a la Convención Internacional sobre los Derechos de las Personas con Discapacidad (BOE 2 de agosto de 2011).

8 Convención sobre los Derechos de las Personas con Discapacidad y Protocolo Facultativo de la Naciones Unidas.

WPS RI-SHUR, nำ2, 2015, vol.1, ISSN: 2387-1768 


\section{WPSReview International on Sustainable \\ Housing and Urban Renewal (RI-SHUR)}

\section{Objetivos}

El objetivo fundamental es promover la mejora de la calidad de los edificios residenciales, involucrando a los usuarios y consumidores en el proceso de mejora continua de la calidad en sus viviendas, tanto nuevas como existentes. La mejora de la calidad en la edificación no debe basarse exclusivamente en las acciones de los profesionales, sino que, al igual que ocurre en otros bienes, es fundamental la participación activa del usuario o consumidor, como demandante de calidad en las viviendas. Para ello, se plantean varios objetivos específicos:

1.- Mejorar el grado de conocimiento de los ciudadanos a la hora de adquirir, alquilar o reformar una vivienda, facilitando la información necesaria para que puedan exigir la mejor calidad siendo conocedores de las mejores prestaciones técnicas.

Con ello se pretende que sean los usuarios a través de sus decisiones e intervención en el mercado quienes obliguen al sector de la edificación a mejorar la calidad de sus productos, limitando la necesidad de que sean las administraciones mediante reglamentaciones cada vez más abundantes y exigentes quienes impongan mejoras que en ocasiones no son solicitadas por los usuarios.

En edificación estamos habituados a fundamentar la mejora de la calidad de los edificios en la normativa y reglamentación nacional, autonómica y local de obligado complimiento por técnicos y empresas. La aplicación de esta normativa garantiza que la calidad de las viviendas actuales, en términos de ahorro de energía, confort acústico, accesibilidad, etc. es mucho mejor que las construcciones de hace 50 años. Sin embargo se ha mantenido ajeno a este proceso a la parte más interesada en él, al ciudadano que compra y usa la vivienda; de este modo, la excesiva reglamentación, a veces tiene como consecuencia un rechazo de los promotores y usuarios, que no entienden por qué invertir y gastar en aspectos que no consideran necesarios. Así convertimos los mínimos exigidos por la normativa en máximos del mercado.

Si bien es indiscutible que la reglamentación debe establecer unos requisitos mínimos obligatorios en todas las edificaciones, es el ciudadano, suficientemente informado y responsable, quien debe exigir al sector mejoras en las calidades de su futura vivienda que mejor se adapten a sus necesidades.

A través de la información y la educación, los usuarios pueden ser los prescriptores de la calidad de sus viviendas, demandando soluciones que WPS RI-SHUR, nำ2, 2015, vol.1, ISSN: 2387-1768 


\section{WPSReview International on Sustainable \\ Housing and Urban Renewal (RI-SHUR)}

reduzcan los consumos energéticos, mejoren la eficiencia, minimicen el impacto medioambiental, reduzcan el consumo de agua, mejoren el confort acústico y la accesibilidad, incorporen energías renovables y materiales ecológicos, etc.

\section{2.- Fomentar la inversión en rehabilitación y mejora del extenso parque de viviendas existentes.}

Mejorar la inversión pública y privada en mantenimiento y rehabilitación, además de permitirnos actualizar un parque de viviendas obsoleto, con la consecuente mejora del confort y del ahorro para los usuarios, tendría un efecto tremendamente positivo sobre el empleo en el sector de la construcción. La actividad de rehabilitación, en este sentido, es una actividad altamente generadora de empleo por unidad de gasto, según estudios realizados para la elaboración del Plan Estatal de Vivienda y Rehabilitación 2009-20129 se estima que por cada millón de euros de inversión en rehabilitación se crean más de 56 empleos, directos, indirectos e inducidos, lo que pone de manifiesto la notable intensidad en términos de empleo que posee la rehabilitación, que además tiene un gran efecto arrastre en las empresas auxiliares y subcontratistas.

Tradicionalmente en España el peso de la conservación y rehabilitación de inmuebles sobre el total de la actividad del sector de la construcción es muy bajo en relación con países de nuestro entorno. La única forma de recuperar parte de la actividad y del empleo perdido en los últimos años por la falta de inversión en obra nueva, es incrementar el nivel de inversión en conservación y rehabilitación de los edificios existentes.

Para ello es necesario, entre otros aspectos, hacer a los usuarios conscientes de las necesidades reales de intervención en el edificio, a través de una correcta evaluación de las necesidades por una parte, y a través de una correcta información sobre sus ventajas. Es decir, hay que transmitirles por qué es importante rehabilitar y mantener los edificios mostrándoles todas las ventajas y beneficios que pueden obtener. $\mathrm{Si}$ rehabilitan adecuadamente su vivienda mejorarán la calidad de la misma aumentando el confort, evitarán el derroche energético reduciendo las facturas, dispondrán de una vivienda más accesible para las personas mayores, y además alargarán la vida de la vivienda e incrementarán su valor económico.

9 Informe sobre la situación del sector de la vivienda en España, Ministerio de vivienda, abril 2010.

WPS RI-SHUR, no2, 2015, vol.1, ISSN: 2387-1768 


\section{WPSReview International on Sustainable \\ Housing and Urban Renewal (RI-SHUR)}

También es necesario ofrecer información al usuario sobre sus obligaciones en el deber de conservación, de las que en muchas ocasiones no es consciente. Los diferentes documentos técnicos que, según las últimas reglamentaciones, el propietario de una vivienda debe disponer, deben ser entendidos como instrumentos que le informan sobre el estado de su vivienda y sobre cómo mejorar la calidad de la misma. Esto no se entiende así en la actualidad, en la que los ciudadanos miran con recelo sus obligaciones y ven como una imposición la necesidad de disponer por ejemplo de la Certificación Energética10 o del Informe de Evaluación del Edificio11 12. Esta situación se da porque nadie les ha explicado la finalidad y utilidad de estos documentos que, bien elaborados por el profesional competente, son herramientas básicas para el adecuado mantenimiento y, en su caso, rehabilitación de las viviendas.

En este sentido, los profesionales del sector tenemos que hacer un esfuerzo divulgativo y educativo hacia los ciudadanos, incidiendo en que la información sobre el estado de las viviendas es el primer paso para mantenerlas y rehabilitarlas mejor

\section{El ciudadano, informado y acompañado por los profesionales, será el motor de la rehabilitación residencial}

\section{Metodología}

En primer lugar es necesario generar la información técnica adecuada y adaptada a los usuarios y consumidores. Para ello en 2013 se puso en marcha la iniciativa "Rehab: + Calidad en tu Vivienda" de la Generalitat Valenciana, desarrollada por el Instituto Valenciano de la Edificación, dirigida a los ciudadanos cuyo material informativo y divulgativo se distribuye fundamentalmente a través de la web www.calidadentuvivienda.es.

En segundo lugar, es necesario generar los mecanismos de distribución de la información que permitan hacer llegar a los ciudadanos toda la información generada. Para ello se han creado redes a través de convenios de colaboración con organismos

10 Real Decreto 235/2013, de 5 de abril, por el que se aprueba el procedimiento básico para la certificación de la eficiencia energética de los edificios.

11 Ley 8/2013, de 26 de junio, de rehabilitación, regeneración y renovación urbanas.

12 Real Decreto 233/2013, de 5 de abril, por el que se regula el Plan Estatal de fomento del alquiler de viviendas, la rehabilitación edificatoria, y la regeneración y renovación urbana, 2013-2016.

WPS RI-SHUR, nำ2, 2015, vol.1, ISSN: 2387-1768 


\section{WPSReview International on Sustainable \\ Housing and Urban Renewal (RI-SHUR)}

y entidades relacionadas, como son ayuntamientos, asociaciones ciudadanas, agentes de la propiedad inmobiliaria, administradores de fincas, etc., con el fin de alcanzar el más alto nivel de difusión entre los usuarios. En el marco de esos convenios se ha realizado acciones divulgativas: jornadas, charlas, distribución de folletos, artículos en revistas, etc.

\section{Desarrollo de la Web www.calidadentuvivienda.es}

La web ofrece toda la información relevante que necesita el ciudadano para mejorar, actualizar y rehabilitar su vivienda, en un lenguaje sencillo y directo. En ella se puede consultar cuáles son las características esenciales que debe incorporar una vivienda si se quiere mejorar su calidad y que incluiría tanto aspectos relacionados con la sostenibilidad, la calidad del aire, ahorro de energía, confort acústico, etc., así como características de los principales elementos del edificio, fachadas, cubiertas, ventanas, etc.

También ofrece información sobre las opciones existentes para llevar a cabo una actualización o rehabilitación de su vivienda, en materia de ahorro energético, comodidad y accesibilidad, presentando soluciones concretas e indicando las principales características de cada una de ellas, así como de los beneficios derivados de su instalación o ejecución.

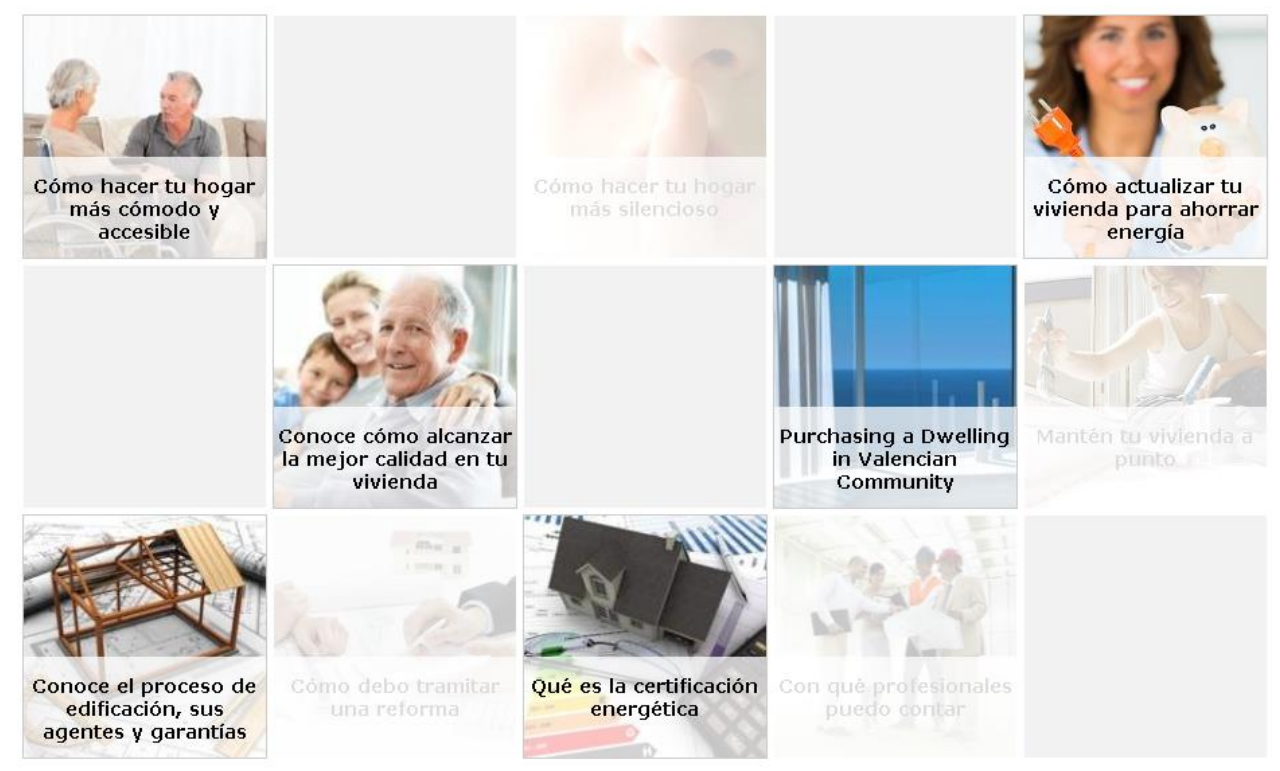

Figura 1: Imagen de la la home de la web www.calidadentuvivienda.es 


\section{WPSReview International on Sustainable \\ Housing and Urban Renewal (RI-SHUR)}

Los principales apartados de la web son:

Mejorar la calidad de la vivienda: a la hora de reformar una vivienda no hay que olvidarse de los aspectos más importantes que mejoran sus condiciones de habitabilidad y la hacen más confortable:

- Cómo ahorrar energía en la vivienda

- Cómo mejorar la accesibilidad

- Cómo evitar los ruidos

- Cómo reducir el impacto medioambiental, ahorrar agua, reciclar.

- Cómo aprovechar los espacios

- Cómo evitar contaminantes en el hogar

Rehabilitación para ahorrar energía: para mejorar el comportamiento energético de las viviendas, la web ofrece una serie de consejos en función del nivel de inversión que se quiere realizar. Es posible ahorrar cambiando los hábitos de consumo, sustituyendo e incorporando pequeños elementos de ahorro, o rehabilitándola energéticamente.

- Cambiar los hábitos de consumo

- Mejorar las ventanas

- Aislar la vivienda

- Renovar las instalaciones, etc.

Rehabilitación para mejorar la accesibilidad: para eliminar barreras arquitectónicas del edificio o de la vivienda, la web ofrece información sobre diferentes tipos de actuación en función del nivel de inversión que se quiere realizar. Se puede adaptar la vivienda y el edificio para facilitar su utilización por las personas mayores o con movilidad reducida.

1. Eliminar barreras arquitectónicas

2. Instalar un ascensor

3. Hacer un baño accessible

4. Colocar elementos de apoyo, etc.

Además se incorporan otros apartados con información complementaria como:

El proceso de la edificación: se presenta al ciudadano cuáles son los agentes que intervienen en todo el proceso, así como de las correspondiente fases que van desde WPS RI-SHUR, n2, 2015, vol.1, ISSN: 2387-1768 


\section{WPSReview International on Sustainable \\ Housing and Urban Renewal (RI-SHUR)}

las iniciales, con el desarrollo del proyecto técnico, hasta las finales relacionadas con la ejecución del edificio y los correspondientes trámites a realizar.

La certificación energética: se explica el objeto del certificado, cuál es su ámbito de aplicación, cómo se puede obtener y cuál es el contenido del mismo. Por último, se dan unas recomendaciones y se presentan unas conclusiones que puedan orientar al ciudadano.

Purchasing a dwelling in the Valencian Community (Comprar viviendas en la Comunidad Valenciana): sección en inglés dirigida principalmente a los ciudadanos extranjeros interesados en la adquisición de una vivienda en la Comunidad Valenciana, dónde pueden encontrar información sobre el sistema de garantías técnicas, legales y administrativas que regula la edificación.

Próximamente se van a incorporar nuevos contenidos a la web, de manera que el usuario pueda encontrar toda la información que necesita para abordar el uso, mantenimiento y reforma de una vivienda.

\section{Mejorar la calidad de la vivienda}

En este apartado la web contiene información tanto de los requisitos que el usuario demanda a una vivienda (ahorro de energía, confort acústico, etc.), como de las características que deben tener los elementos constructivos (fachadas, huecos exteriores, etc.) para alcanzar determinados niveles de calidad.

En base a una serie de preguntas concretas sobre calidad: ¿Cómo elegir las carpinterías de las ventanas?, ¿Cómo debe ser un baño accesible?, etc. se desarrollan temas técnicos de calidad en la edificación adaptados al vocabulario y conocimiento del público en general.

La web ofrece diferentes niveles de información para adaptarse a las diversas necesidades de los usuarios. Partiendo de preguntas específicas se ofrece una información concreta y reducida, a partir de la cual el usuario puede obtener información más detallada según su grado de interés. Existen tres niveles de información: preguntas concretas "¿Cómo elegir las carpinterías de las ventanas?"; requisitos de calidad "Ahorro de energía", y elementos constructivos "Ventanas" Los aspectos de calidad que se desarrollan en la web son:

-Ahorro de energía: El comportamiento energético de una vivienda depende de la orientación del edificio, el aislamiento térmico de su envolvente, las características de WPS RI-SHUR, nำ2, 2015, vol.1, ISSN: 2387-1768 


\section{WPSReview International on Sustainable \\ Housing and Urban Renewal (RI-SHUR)}

sus ventanas, la eficiencia de sus instalaciones térmicas, la eficiencia de la iluminación, etc... Es importante que el usuario tenga información adecuada sobre estos aspectos pues van a determinar que la vivienda sea confortable térmicamente y de baja o moderada demanda energética, alcanzando ahorros de energía de hasta un $30 \%$ y reduciendo las emisiones de $\mathrm{CO} 2$ hasta un 35\%, cuya repercusión influye directamente en un ahorro para el consumidor.

-Confort acústico: el usuario es cada vez más exigente respecto a la calidad acústica de su vivienda, y valora todas aquellas medidas que favorezcan un buen aislamiento. Mejoras en los elementos constructivos pueden incrementar el aislamiento frente a los ruidos producidos en el exterior del edificio y en las viviendas contiguas, y frente a los ruidos producidos por las instalaciones. De esta forma las viviendas ofrecen un ambiente saludable y favorecen el descanso.

-Calidad del aire interior: la calidad del aire de la vivienda afecta a la salud de sus ocupantes, y depende de factores como la ubicación y orientación del edificio, las posibles fuentes contaminantes de los materiales utilizados en la construcción, el exceso de humedad interior, etc. Para garantizar calidad del aire interior es necesario que los edificios dispongan de medios para que se puedan ventilar adecuadamente, y garanticen la renovación suficiente del aire.

-Sostenibilidad: es importante que, en la medida de lo posible, el edificio incorpore medidas que protegen el medio ambiente: el ahorro de agua, la utilización de materiales sostenibles, la correcta gestión de los residuos y la reducción en la demanda de recursos. Existen sistemas de ahorro de agua en el mercado, fáciles de incorporar en la vivienda, que producen ahorros en el consumo de agua de hasta un $35 \%$ : Si el usuario tiene información sobre estas medidas puede demandarlas para su vivienda.

-Accesibilidad: para que los edificios de viviendas puedan ser utilizados por todas las personas, incluidas las personas con discapacidad, los recorridos hasta la vivienda y en el interior de la misma deben cumplir unas condiciones que permitan su uso de forma autónoma y cómoda. Con medidas sencillas en el interior de la vivienda, se puede conseguir que una unidad de vida (estar, cocina, baño y dormitorio) sea accesible. De esta forma, podemos garantizar el uso de nuestra vivienda por personas mayores o frente a una situación transitoria de movilidad reducida.

-Funcionalidad: para que en el interior de la vivienda puedan desarrollarse todas las funciones para las que está destinada, la vivienda debe contar con los espacios WPS RI-SHUR, no2, 2015, vol.1, ISSN: 2387-1768 


\section{WPSReview International on Sustainable \\ Housing and Urban Renewal (RI-SHUR)}

necesarios para ello. Estos espacios deben reunir las condiciones dimensionales adecuadas, y contar con las instalaciones, acabados y equipamientos necesarios.

Como la calidad de la vivienda en estos aspectos depende de la calidad de cada uno de los elementos constructivos y espacios del edificio, en este apartado se amplía la información técnica de los mismos desarrollando una ficha de calidad para cada uno de los elementos determinantes de la calidad: Fachadas, Ventanas, Cubiertas, Particiones, Revestimientos, Instalaciones, Equipamiento, Baños y Cocinas.

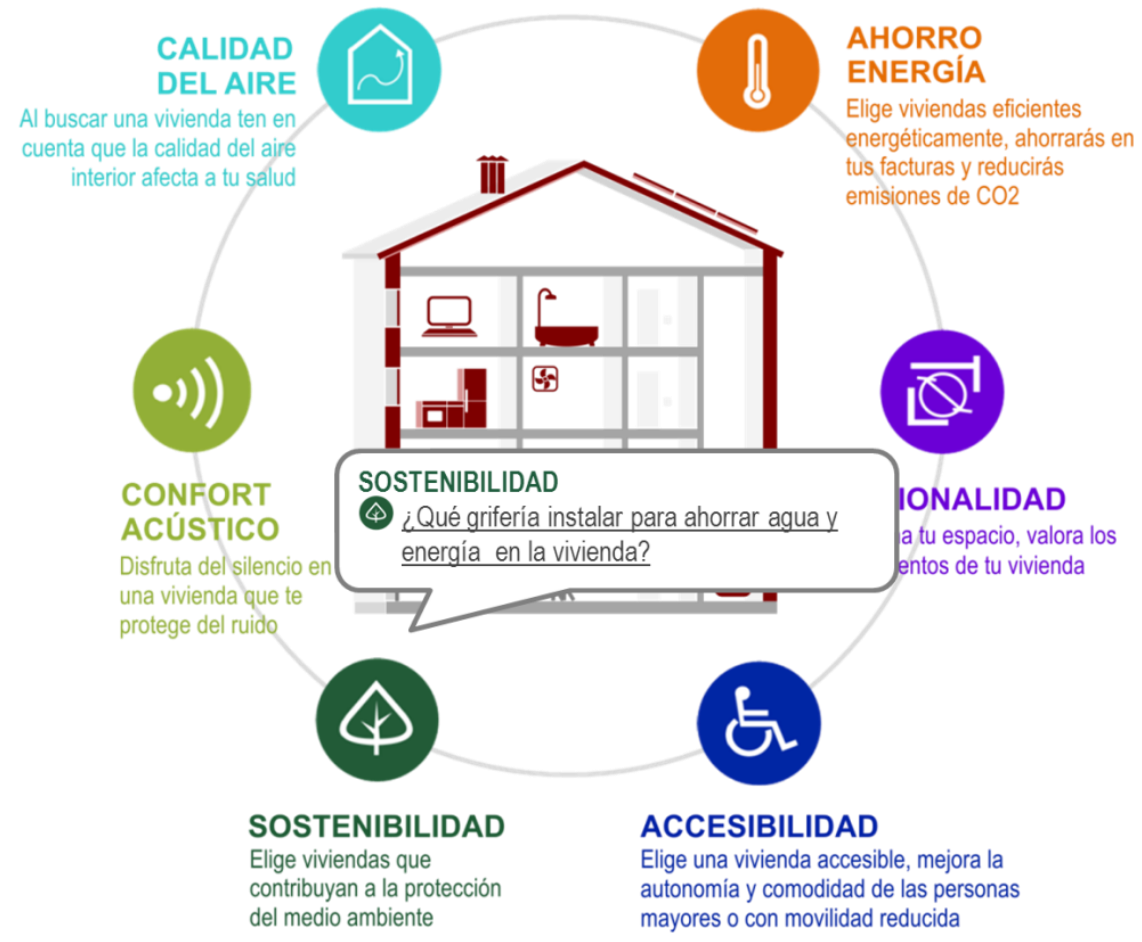

Figura 2: Imagen del apartado Mejorar la calidad de la vivienda de la web

\section{Rehabilitación para ahorrar energía}

En este apartado la web contiene información sobre cómo reducir el consumo de energía de la vivienda. El usuario puede acceder a esta información siguiendo un criterio económico en función del nivel de inversión que quiere realizar, o bien partiendo del elemento de la vivienda en el que quiere intervenir: 


\section{WPSReview International on Sustainable \\ Housing and Urban Renewal (RI-SHUR)}

Nivel de inversión: las medidas de ahorro de energía se organizan en función del nivel de inversión económica que mejor se adapte a nuestras necesidades, van desde medidas relacionadas con cambios en los hábitos de consumo, medidas que requieren pequeñas inversiones incorporando elementos de ahorro, o bien, medidas de renovación y rehabilitación que supone una mayor inversión pero con las cuales los ahorros conseguidos son muy significativos.

Elementos a intervenir: los elementos del edificio o de la vivienda que se pueden renovar o mejorar: instalación de calefacción y refrigeración, aislamiento térmico de cubiertas y fachadas, etc.
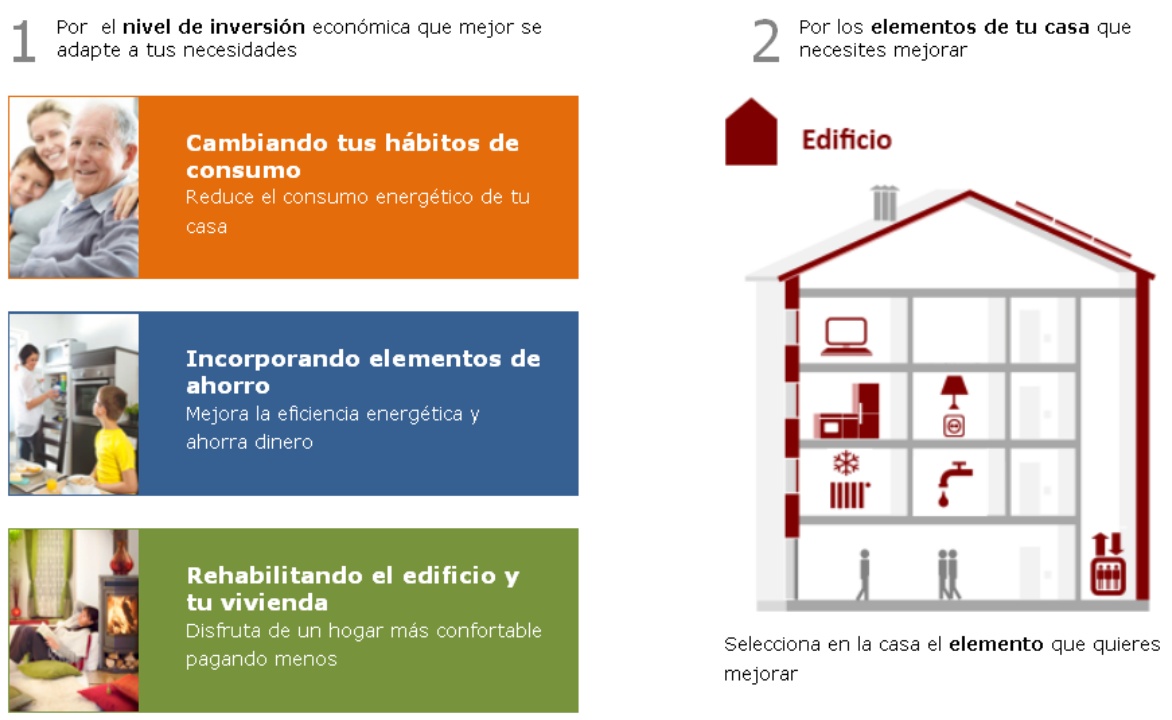

Figura 3: Imagen del apartado Ahorra energía en tu vivienda de la web

Por tanto, la información de este apartado está distribuida en 40 fichas estructuradas en una serie de apartados comunes. En el siguiente cuadro podemos ver el cruce entre nivel de inversión y elementos del edificio que da lugar a las fichas desarrolladas.

Tabla 1: Fichas según nivel de inversión y elementos a intervenir

\begin{tabular}{|c|c|c|c|}
\hline & $\begin{array}{l}\text { Cambio } \\
\text { de hábitos }\end{array}$ & $\begin{array}{l}\text { Elementos } \\
\text { de ahorro }\end{array}$ & $\begin{array}{l}\text { Renovación } \\
\text { o rehabilitación }\end{array}$ \\
\hline Iluminación y electricidad & 2 & 3 & \\
\hline Electrodomésticos & 3 & 1 & \\
\hline
\end{tabular}

WPS RI-SHUR, n2, 2015, vol.1, ISSN: 2387-1768 
WPSReview International on Sustainable

Housing and Urban Renewal (RI-SHUR)

\begin{tabular}{|r|l|l|l|}
\hline Domótica & & 1 & \\
\hline Agua caliente & 1 & 2 & \\
\hline Climatización & 2 & 2 & 2 \\
\hline Ascensor & & 1 & \\
\hline Ventanas & 2 & 3 & 3 \\
\hline Fachadas y cubiertas & & 1 & 8 \\
\hline Energías renovables & & & 1 \\
\hline Edificio & 1 & & 1 \\
\hline
\end{tabular}

Cada una de las fichas se estructura en los siguientes apartados:

-Título de la ficha: se trata de una pregunta concreta sobre cómo ahorrar, por ejemplo, ¿Cómo ahorrar energía aislando térmicamente las fachadas de su edificio?

-¿Cuál es el problema?: se explica el problema debido al cuál se plantea la intervención.

-¿Cómo puedo solucionarlo?: se proponen diferentes alternativas para solucionar el problema planteado en el apartado anterior.

-¿Qué ahorro puedo obtener?: se establece un intervalo del ahorro que se puede obtener mediante la aplicación de las medidas planteadas, y se definen los factores de los cuales depende que el ahorro sea mayor o menor dentro del intervalo aportado. Para estimar estos ahorros se ha realizado un análisis energético en diferentes tipologías edificatorias y en función de la zona climática incorporando las medidas de mejora planteadas.

-Info: contiene información complementaria de la medida planteada que variará según la ficha, consejos, amortizaciones, ventajas e inconvenientes, coste de la instalación por vivienda, ahorro en el gasto energético por año, etc. 
WPSReview International on Sustainable

Housing and Urban Renewal (RI-SHUR)
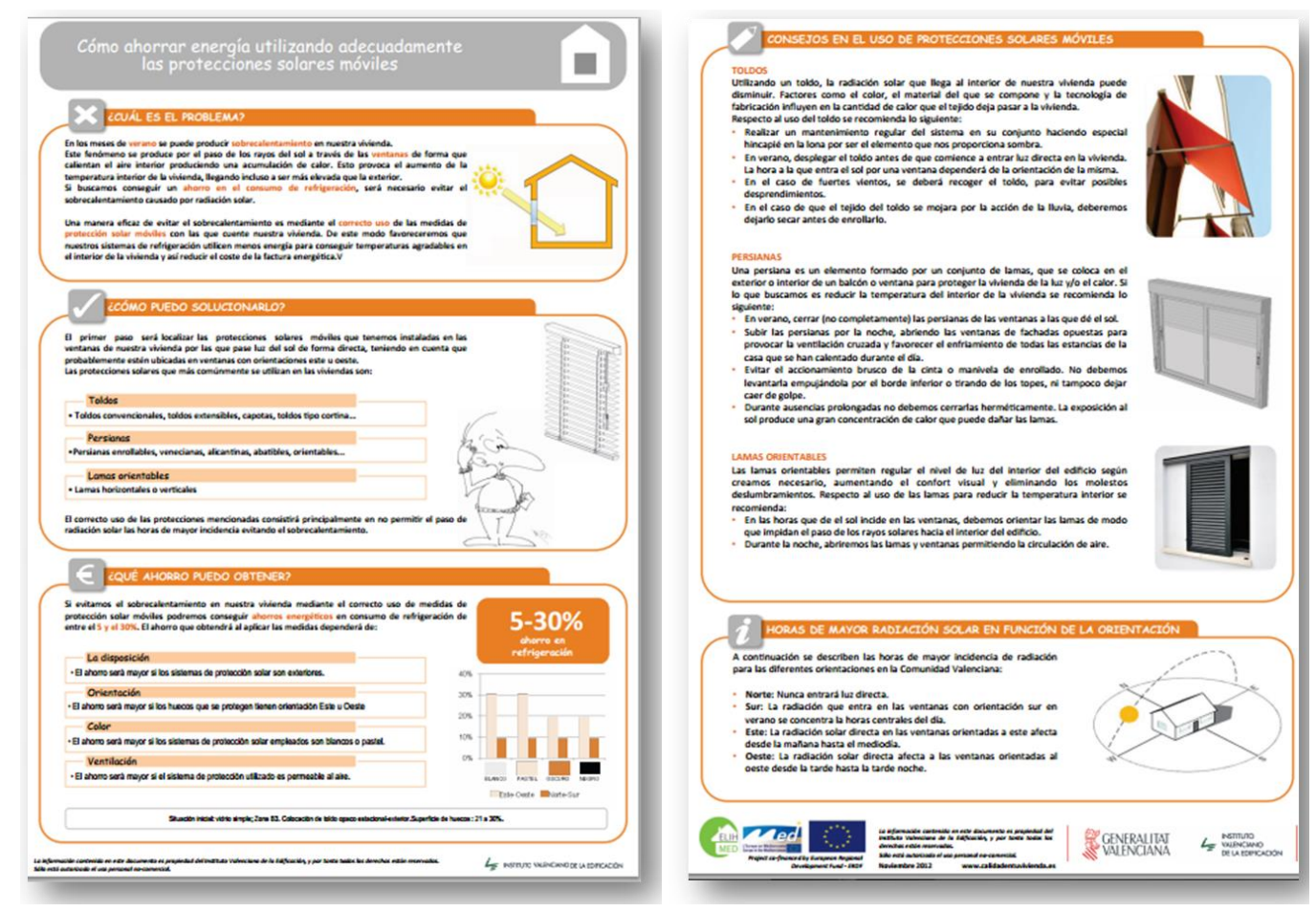

Fig. 10: Imagen de las fichas descargables de la web

\section{Rehabilitación para mejorar la accesibilidad:}

Se ofrece información sobre cómo eliminar barreras arquitectónicas del edificio o de la vivienda y permitir a las personas mayores y con movilidad reducida un uso más cómodo. Al igual que en el apartado anterior el usuario puede acceder a esta información siguiendo un criterio económico en función del nivel de inversión que quiere realizar, o bien partiendo del elemento de la vivienda en el que quiere intervenir. Se trata de un un conjunto de 10 fichas con una estructura común de apartados: ¿cuál es el problema?; ¿cómo puedo solucionarlo?; info complementaria.

\section{Mecanismos de distribución de la información}

\section{-Creación de redes de usuarios}

Creación de una red de usuarios de viviendas a través de la firma de convenios de colaboración con entidades representantes e intermediarias de los mismos, y diferentes entidades relacionadas con el sector de la vivienda. La red de usuarios será el mecanismo que por un lado permitirá dar a conocer al gran público la web, y por

WPS RI-SHUR, nำ2, 2015, vol.1, ISSN: 2387-1768 


\section{WPSReview International on Sustainable \\ Housing and Urban Renewal (RI-SHUR)}

otro permitirá identificará las necesidades reales de la demanda, averiguando dificultades y mejoras que permitan adaptar el mercado a nuevos enfoques.

El objetivo de los convenios es colaborar en la difusión de la web www.calidadentuvivienda.es y de su fondo documental, poniendo a disposición de las entidades firmantes el material desarrollado y el conocimiento de los técnicos del IVE para la realización de acciones de difusión y sensibilización realizadas conjuntamente.

Actualmente el instituto ha formalizado convenios con entidades de la siguiente índole:

- Asociaciones de consumidores y usuarios

- Asociaciones de amas de casa

- Asociaciones de vecinos

- Colegios de Administradores de Fincas

- Colegios de Agentes de la Propiedad Inmobiliaria

- Ayuntamientos y entes públicos

- Asociaciones profesionales y empresariales del sector de la construcción

\section{Difusión de la información}

En el marco de los convenios, se han realizado una serie de acciones de difusión:

-Charlas y jornadas dirigida tanto a profesionales y empresarios del sector como a consumidores y usuarios, organizadas en colaboración con las entidades firmantes de los convenios.

-Diseño e impresión de folletos informativos sobre la web www.calidadentuvivienda.es que se han distribuido entre las entidades firmantes del convenio y los asistentes a los actos y jornadas celebrados.

-Comunicación a los ayuntamientos, con el fin de que conozcan la iniciativa y puedan colaborar en su difusión entre los ciudadanos de su municipio. Junto a esta comunicación se les envía carteles promocionales y folletos de la iniciativa.

-Artículos en revistas de consumidores y profesionales.

-Links a la web de calidad desde las diferentes web de las entidades firmantes. 


\section{WPSReview International on Sustainable \\ Housing and Urban Renewal (RI-SHUR)}

\section{Resultados y conclusiones}

Los objetivos se plantean a medio y largo plazo, pero hay una serie de indicadores que pueden servirnos de referencia de los resultados obtenidos, desde enero de 2013 hasta mayo de 2014:

- 80.000 visitas a la página web.

- 53 Convenios de colaboración suscritos.

- 30 Charlas y jornadas con un total de más de 1.300 asistentes.

- 5.000 folletos distribuidos.

- 129 comunicados a ayuntamientos de más de 10.000 habitantes, con envío de carteles informativos.

Las principales conclusiones de esta iniciativa son:

- Mejorar la calidad de las viviendas implica un esfuerzo de colaboración que involucre a todos los agentes del sector: empresa, industria, profesionales y usuarios. Tras años de regulación normativa y participación de los sectores empresariales y profesionales, es necesario un gran esfuerzo con el objeto de implicar al usuario final de la vivienda en el proceso de mejora de la calidad. Para que esta implicación sea efectiva es necesario que el usuario disponga de la información adecuada y necesaria para que sus decisiones tengan en cuenta aspectos esenciales de la calidad de las viviendas, y no exclusivamente aspectos estéticos y aparentes. Para ello se ha desarrollado la web calidadentuvivienda.es que sirve de plataforma y soporte de toda la información que se quiere pone a disposición de los ciudadanos. Se trata de una plataforma cuyo contenido es de ámbito nacional, y actualmente está disponible en castellano.

- Llegar al consumidor final es una tarea compleja que debe plantearse a medio largo plazo. Las campañas publicitarias que serían necesarias son inviables, pues requieren unos recursos económicos que no se disponen en la actual situación. La alternativa es crear redes de distribución de la información a través de la colaboración entre entidades que representan a los consumidores (asociaciones de usuario, de vecinos); entidades profesionales cercana a ellos (administradores de fincas, agentes de la propiedad inmobiliaria, técnicos); entidades públicas cercanas a los ciudadanos como son los ayuntamientos; etc. La acciones que se han realizado para la creación de estas redes y la utilización de las mismas para distribuir y divulgar la información, se han desarrollado fundamentalmente en la Comunidad Valenciana. En un futuro sería deseable que se extendieran al ámbito nacional, mediante la colaboración con entidades con implantación en todo el país, o en otras comunidades autónomas.

WPS RI-SHUR, n2, 2015, vol.1, ISSN: 2387-1768 


\section{WPSReview International on Sustainable}

\section{Housing and Urban Renewal (RI-SHUR)}

-Si se logra que los ciudadanos adquieran una cultura de la calidad en edificación exigirán esa calidad a la hora de comprar o alquilar una vivienda, empujando al mercado a competir en este sentido. Además, si toman conciencia de las mejoras y ahorros que se pueden obtener se estimulará la inversión en rehabilitación y, como consecuencia, se puede impulsar la actividad en el sector de la construcción con la consiguiente repercusión positiva en la economía. Todo esto supone una reducción de emisiones de $\mathrm{CO} 2$ necesaria para cumplir los objetivos europeos, mejoras de la calidad de vida y confort de los ciudadanos en sus viviendas, y mejoras en materia de accesibilidad adaptando los edificios y viviendas a sus ocupantes.

\section{Correspondencia}

Nombre y Apellido: CARMEN SUBIRÓN RODRIGO

Teléfono: + 34963986505

Fax: + 34963986504

E-mail: csubiron@five.es 